\title{
Adherence to Antihypertensives in Patients With Comorbid Condition
}

\author{
Zahra Saadat ${ }^{1}$; Farahnaz Nikdoust ${ }^{2}$; Hossein Aerab-Sheibani ${ }^{3}$; Mostafa Bahremand ${ }^{4, *}$; \\ Elham Shobeiri ${ }^{5}$; Habibollah Saadat ${ }^{1}$; Yashar Moharramzad $^{1}$; Donald E. Morisky ${ }^{6}$ \\ ${ }^{1}$ Department of Cardiology, Cardiovascular Research Center, Shahid Beheshti University of Medical Sciences, Tehran, IR Iran \\ ${ }_{3}^{2}$ Department of Cardiology, Shariati Hospital, Tehran University of Medical Sciences, Tehran, IR Iran \\ 3 Department of Cardiology, Shahid Sadoughi University of Medical Sciences, Bafgh, Kermanshah, IR Iran \\ 4Department of Cardiology, Shahid Sadoughi University of Medical Sciences, Bafgh, Kermanshah, IR Iran \\ 5 Department of Cardiology, School of Medicine, Kermanshah University of Medical Sciences, \\ ${ }_{6}^{5}$ Department of Radiology, Kermanshah University of Medical Sciences, Kermanshah, IR Iran
${ }_{\text {Department of Community Health Sciences, UCLA Fielding School of Public Health, Los Angeles, USA }}$ \\ ${ }^{*}$ Corresponding author: Mostafa Bahremand, Department of Cardiology, School of Medicine, Kermanshah University of Medical Sciences, Kermanshah, IR Iran. Tel: +98-8338360014, \\ E-mail:dr-bahremand@kums.ac.ir
}

Received: May 10, 2015; Revised: June 28, 2015; Accepted: July 11, 2015

\begin{abstract}
Background: Comorbidity has been noted as a potential barrier to proper adherence to antihypertensive medications.
Objectives: We decided to investigate whether comorbidity could significantly affect adherence of Iranian patients with hypertension to their medication regimen.

Patients and Methods: Two hundred and eighty consecutive hypertensive patients were interviewed in 4 cities of Iran. The 8-item Morisky medication adherence scale (MMAS-8) (validated in Persian) was used to assess medication adherence. This scale determines adherence by scores as lower than 6 (low adherence), 6 or 7 (moderate adherence), and 8 (high adherence). Comorbidity was considered as any concomitant medical condition, which necessitates the patient to take medicine for a minimum of 6 months prior to the interviews. Results: The most common comorbid conditions were ischemic heart disease (65 patients, 23.2\%), diabetes mellitus (55 patients, 19.6\%), and dyslipidemia (51 patients, 18.2\%). Mean ( \pm SD) MMAS-8 score in comorbid group was $5.68( \pm 1.85)$ and in non-comorbid hypertensive patients, it was $5.83( \pm 1.91)(\mathrm{P}=0.631)$. Mean $( \pm \mathrm{SD})$ number of comorbidities was $1.53( \pm 0.75)$ in low adherence group compared to 1.54 $( \pm 0.77)$ in moderate/high adherers $(\mathrm{P}=0.98)$. With increasing the number of comorbid diseases, the proportion of patients with high adherence decreased successively from $20 \%$ in those with no comorbid disease to $14.1 \%$ in those with one or two comorbid conditions, and finally $11.1 \%$ in those with 3 to 5 comorbid conditions.

Conclusions: With increasing the number of comorbid conditions, the proportion of patients with high adherence decreases. In our opinion, this finding is a useful clinical note for healthcare providers when managing patients with hypertension who have other medical problems at the same time.
\end{abstract}

Keywords: Hypertension; Comorbidity; Iran; Medication adherence; Antihypertensive agents

\section{Background}

Undoubtedly, one of the factors in controlling high blood pressure (BP) is good adherence to medication(s) prescribed. Adherence to (compliance with) the medication has been described as active involvement of patients in taking medications prescribed to achieve an acceptable therapeutic result $(1,2)$. This is of so paramount clinical importance that even some experts believe that maximizing adherence is more important in achieving good BP control compared to selecting a special drug class or regimen in the era of promising evidence of new classes of antihypertensives (3).

In essence, medication adherence is a complex phenomenon and discrete factors can influence on it. One of these contributing factors (which we think the relevant data about it are suboptimal) is the presence of a concomitant medical condition (i.e., comorbidity) (4). Previous pertinent studies reported conflicting results. Some reported that adherence to antihypertensives decreases when co- morbidity exists $(5,6)$. In other words, existence of multiple medical conditions has been viewed as a barrier to good medication adherence and prompts nonadherence. For instance, it was reported that medication adherence, measured by medication possession ratio days' supply of the drug dispensed in 1-year follow-up, was $80 \%$ in $72.3 \%$ of individuals with hypertension (HTN) alone, which was better when compared to hypothyroidism comorbidity (68.4\%) or gout comorbidity (36.8\%) (7). Comorbidities are generally addressed as barriers for patients with hypertension and putting them into consideration when assessing the patients' adherence (3). In addition to physical illnesses, psychiatric conditions such as depressive symptoms have also been reported to adversely affect the adherence to antihypertensives (4) and cause poor BP control (8).

In contrast, other authors found good adherence to antihypertensives in diabetic patients who were taking these drugs, a finding which was discovered in North

Copyright (C) 2015, Nephrology and Urology Research Center. This is an open-access article distributed under the terms of the Creative Commons Attribution-NonCommercial 4.0 International License (http://creativecommons.org/licenses/by-nc/4.0/) which permits copy and redistribute the material just in noncommercial usages, provided the original work is properly cited. 
American countries (9-12). Another study from Scotland also did not find any relationship between the number of comorbidities and adherence (13).

\section{Objectives}

Considering the aforementioned facts, the scope of this study was to explore the hypothesis that whether noncomorbid patients with hypertension have better adherence to medication compared to comorbid patients and how presence of an additional medical condition, specific disease, and the number of comorbidities affect the adherence of patients with high BP to the prescribed antihypertensive drugs. We believe that the findings of this study will be of splendid benefit for health care providers who deal with patients suffering from HTN and at least one concomitant medical condition in order to implement the findings in attaining good BP control.

\section{Patients and Methods}

This study with cross-sectional design method was conducted from August 2014 to December 2014. Sampling of the patients was done at several healthcare centers, including cardiology outpatient clinic of a university hospital, private cardiology, general practice offices, and pharmacy. These centers were located in Tehran, Karaj, Kermanshah, and Bafgh. Inclusion criteria consisted of patients aged more than 18 years with HTN under treatment. The interviews with the patients were done face to face by the research team members.

To determine the adherence to antihypertensives, the Persian version of the MMAS-8 (the 8-item Morisky Medication Adherence Scale) (validated by our team in a previous study) was administered to the patients. The MMAS-8 was designed to assess how well the patients adhere to their medications (Appendix 1) (14). As shown in Appendix 1, it has 8 questions. For questions 1 to 7 , the answer options are "yes" or "no". The eighth question is a Likerttype question. The overall score of this scale is from 0 to 8 . The categorization of patients considering their score is as follows: less than 6 (low adherence), 6 or 7 (moderate adherence), and 8 (high adherence). This scale showed a sensitivity of $93 \%$ in finding those individuals with poor adherence (15). It was also demonstrated to be significantly associated with antihypertensive drug pharmacy refill adherence (16). In our previous study on 200 Iranian patients suffering from HTN, we determined the Persian version of the MMAS- 8 to be a valid and reliable tool with the Cronbach a coefficient of 0.697 and test-retest reliability of 0.940 (17).

Besides MMAS-8, we employed a data-gathering form to collect demographic information as well as variables about other diseases or medications the patients reported or took at the time of study. Comorbidity was considered as any concomitant medical condition, which necessitates the patient to take medicine for at least 6 months before the interview time to control symptoms and signs of the disease or impaired laboratory data (e.g., blood glucose, lipid profile, thyroid function tests). Other medical condition(s) could be secondary to HTN or unrelated to that. Chronic Kidney Disease (CKD) was defined in patients with documents (laboratory, nephrologist, or discharge reports) of impaired renal function and decreased glomerular filtration rate (GFR) so severe that patients had to receive medications (e.g., for anemia) and or were receiving hemodialysis (18).

First, the MMAS was completed by the patients. After completing it, the checklist data was completed. The variables of the checklist consisted of demographic data (age, gender, weight, height, occupation, educational level), HTN duration, medications prescribed for HTN, other comorbidities, medications other than antihypertensives, and control of hypertension during the last 6 months by a healthcare provider. At the end, BP of the patients was measured. For this purpose, sphygmomanometer was used to measure BP on the left arm in sitting position. The patients were asked to sit relaxed and not to smoke for half an hour before BP measurement.

\subsection{Statistical Analyses}

Descriptive indexes, including frequency, percentage, mean, and standard deviation were used to express data. Adherence level was compared between the groups with the Chi-square test. The MMAS-8 scores were compared between groups by the Mann-Whitney U test. All analyses were done by the SPSS software for Windows (ver. 16.0). Significance level was set at 0.05 .

\subsection{Ethics}

After describing the purpose of the study and gathering information, verbal consent was obtained from the patients.

\section{Results}

Mean $( \pm$ SD) age of the participants was $60.3( \pm 10.0)$ years. Mean $( \pm$ SD) duration of HTN was $7.2( \pm 5.9)$ years (range, 6 months to 40 years). Table 1 summarizes demographic characteristics of the sample.

General practitioners (67 patients, 23.9\%) and cardiologists (65 patients, $23.2 \%$ ) were the most reported physicians whom the patients visited to control their high BP. Others reported visiting internists (24 patients, 8.6\%), nephrologists ( 9 patients, $3.2 \%$ ), or more than one physician to check their $\mathrm{BP}$ (115 patients, $41.0 \%)$.

Nearly half of the patients (127 patients, $45.3 \%$ ) were taking one class of antihypertensive medication for their condition. Over one-fourth of the patients, 74 (26.4\%) were prescribed angiotensin receptor blockers, the most prevalent medication used.

Mean ( \pm SD) overall MMAS-8 score was 5.75 ( \pm 1.88 ). About half of the sample (139 patients, $49.6 \%$ ) showed low adherence to antihypertensives. Ninety-five patients (33.9\%) had moderate adherence and 46 patients (16.4\%) showed high adherence level. 
Saadat Z et al.

Table 1. Demographic Characteristics of 280 Patients with Hypertension

\begin{tabular}{lc}
\hline Demographic Characteristics & Frequency (\%) \\
\hline Gender & \\
Male & $118(42.1)$ \\
Female & $162(57.9)$ \\
Age, $\mathbf{y}$ & \\
$\quad \leq 50$ & $51(18.2)$ \\
$>50$ & $229(81.8)$ \\
BMI, kg/m 2 & \\
$<25$ & $76(27.1)$ \\
\hline$\geq 25$ & $204(72.9)$ \\
Education & \\
\hline Lower than high school diploma/illiterate & $164(58.5)$ \\
\hline High school diploma & $71(25.4)$ \\
\hline Academic degree & $45(16.1)$ \\
Occupation & \\
\hline Market trader/self-employed & $73(26.1)$ \\
\hline Clerk/military & $41(14.6)$ \\
\hline Housewife & $119(42.5)$ \\
\hline Retired/unemployed & $47(16.8)$ \\
\hline Current smoker & $49(17.5)$ \\
\hline Insurance coverage & $246(87.8)$ \\
\hline Residence place & \\
\hline Urban & $273(97.5)$ \\
\hline Rural & $7(2.5)$ \\
\hline
\end{tabular}

Of 280 patients, 160 patients (57.1\%) had at least one comorbid condition. However, 120 patients (42.9\%) did not have any comorbidity. Ninety-six patients (34.2\%) had one comorbid disease, 46 patients $(16.4 \%$ ) had 2 comorbid diseases, and 18 patients (6.4\%) had 3 diseases or more with HTN. The most common comorbid condition was ischemic heart disease (65 patients, 23.2\%) with or without history of coronary artery bypass grafting or percutaneous coronary intervention, followed by diabetes mellitus (55 patients, 19.6\%), dyslipidemia (51 patients, $18.2 \%$ ), psychiatric conditions (8 patients, $2.8 \%$ ), CKD (7 patients, $2.5 \%$ ), as well as gout and hypothyroidism (each was seen in 6 patients, 2.1\%). Other conditions (congestive heart failure, osteoporosis, chronic obstructive pulmonary disease, malignancies, chronic low back pain, anemia, benign prostatic hyperplasia, osteoarthritis, and so on) had frequencies of 5 patients or fewer.

Mean ( \pm SD) MMAS-8 score in comorbid group was 5.68 $( \pm 1.85)$ and in non-comorbid hypertensives was 5.83 ( \pm 1.91) $(\mathrm{P}=0.631)$. Table 2 presents the comparison of adherence levels (low vs. moderate/high adherence) in terms of comorbidity and its mean number. As shown, no significant difference was detected between low adheres and moderate/good adherers regarding these two variables.

In Table 3, comparison of 3 most common comorbid conditions between low adherers and moderate/high adherers is depicted. Similar to Table 2, no significant difference was observed regarding these 3 comorbid conditions between low and moderate/high adherence levels.

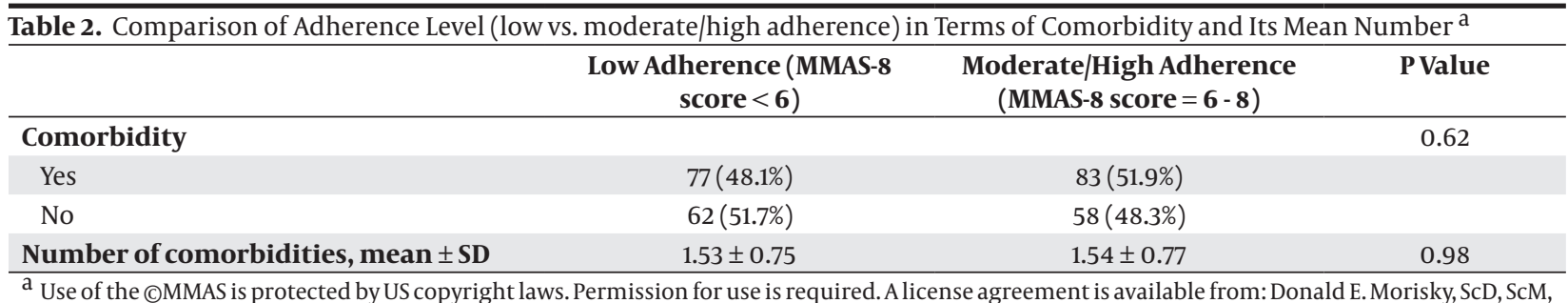

a Use of the @MMAS is protected by US copyright laws. Permission for use is required. Alicense agreement is available from:Donald E. Morisky, ScD, ScM,
MSPH, Professor, Department of Community Health Sciences, UCLA Fielding School of Public Health, 650 Charles E. Young Drive South, Los Angeles, CA.

Table 3. Anti-Hypertensive Adherence Level Status Considering the 3 Most Common Comorbid Conditions Reported in the Sample of 280 Patients With Hypertension ${ }^{\mathrm{a}, \mathrm{b}}$

\begin{tabular}{|c|c|c|c|}
\hline & $\begin{array}{c}\text { Low Adherence (MMAS-8 } \\
\text { Score }<6 \text { ), N. (\%) }\end{array}$ & $\begin{array}{l}\text { Moderate/High Adherence } \\
\text { (MMAS-8 score = 6-8), N. (\%) }\end{array}$ & P Value \\
\hline $\mathrm{IHD} \pm \mathrm{CABG} / \mathrm{PCI}{ }^{\mathrm{a}, \mathrm{b}}$ & & & 0.48 \\
\hline Yes & $35(53.8)$ & $30(46.2)$ & \\
\hline No & $104(48.4)$ & $111(51.6)$ & \\
\hline Dyslipidemia & & & 0.87 \\
\hline Yes & $26(51)$ & $25(49)$ & \\
\hline No & $113(49.3)$ & $115(50.7)$ & \\
\hline DM & & & 0.22 \\
\hline Yes & $23(41.8)$ & $32(58.2)$ & \\
\hline No & $116(51.6)$ & $109(48.4)$ & \\
\hline
\end{tabular}

a Abbreviations: CABG, Coronary artery bypass grafting; DM, Diabetes mellitus; PCI, Percutaneous coronary intervention.

b Use of the @MMAS is protected by US copyright laws. Permission for use is required. A license agreement is available from: Donald E. Morisky, ScD, ScM, MSPH, Professor, Department of Community Health Sciences, UCLA Fielding School of Public Health, 650 Charles E. Young Drive South, Los Angeles, CA. 
Of 7 patients with CKD, 3 patients had only CKD; 1 patient had CKD and IHD. One patient had CKD and gout. Two patients had DM and IHD in addition to CKD. Mean (SD) MMAS-8 score in this group was 6.21 (1.72); range 3 - 8. Two patients (one with CKD and another with CKD plus gout) had good adherence (i.e., score of 8 ). One patient with just CKD had poor adherence (score of 3 ). Others had moderate adherence level.

Figure 1 depicts adherence level (low, moderate, and high) with respect to the number of comorbidities. As seen, with increasing the number of comorbid conditions, the proportion of high adherers decreased. In fact, high level of adherence (i.e., MMAS-8 score $=8$ ) was calculated in patients without comorbidity as $20.0 \%$. It was $14.1 \%$ for those with one or two comorbid conditions, and $11.1 \%$ for those with 3 - 5 comorbid conditions.

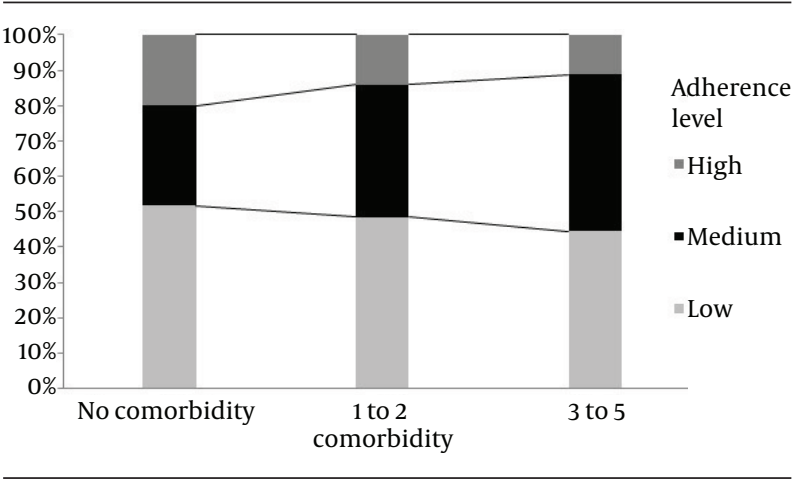

Figure 1. Antihypertensive Adherence Level (Low, Moderate, and High) and Number of Comorbid Conditions

\section{Discussion}

Adherence studies in Iran are extremely insufficient. There are few relevant studies targeting patients with HTN, however their design, quality, and methods used to define adherence are questionable (19). We did not find any previous study to address patient and practice-related as well as adherence report in Iranian hypertensive patients with multiple medical conditions. Adherence studies in such patients can be even more important considering the fact that cardiovascular events increase several times when a hypertensive patient has concomitant conditions such as diabetes or dyslipidemia (6).

According to the obtained findings, comorbidities that necessitated regular medication consumption did not affect the patients' compliance with antihypertensive. Neither the number of comorbidities, nor their specific types (IHD, DM, and dyslipidemia) were found to adversely affect the adherence level. The presence of comorbidity and its influence on adherence can be viewed from 2 aspects.

One is related to the nature of the comorbidity itself and the other relates to the medication taken by the patient for the comorbidity. The clinicians should not ignore the second view. For example, concomitant use of antihypertensive and lipid-lowering therapy was associated with poor adherence and about $45 \%$ were demonstrated to be adherent; the figure declined to $36 \%$ at 1-year follow-up (20). As mentioned earlier, the results of former studies about the role of comorbidity are heterogeneous. This may be related to the fact that adherence to medication is a complex phenomenon and various factors, including knowledge and attitude of patients towards HTN, health care system issues, doctor-patient relationship, and the role of pharmacists all have significant impact on adherence and its improvement $(5,21-23)$.

In an American study (6) to evaluate adherence in hypertensive patients with DM (using proportional days covered (PDC) for both oral DM and HTN medications), the authors reported that preexisting disease, older age, higher number of medications, and doctor-related variables (e.g., prescription for both DM and HTN medications provided by a single doctor) were significant factors in determining multiple medication adherence. In contrast, Chapman et al. found that taking fewer medications was a factor associated with better adherence in patients taking medications for HTN and dyslipidemia (20). In another study conducted on more than 700,000 patients, using mean medication possession ratio to determine adherence, it was shown that adherence decreased with the presence of any of the 6 comorbid conditions, including hypothyroidism, type $2 \mathrm{DM}$, seizure disorders, hypercholesterolemia, osteoporosis, and gout (7).

\subsection{Strengths and limitations}

Given that the presented data were collected from different settings, including outpatient cardiology clinic of university hospital, private cardiology and general practice offices, and pharmacy at different parts of Iran, the sample may be a good representative of Iranian population and the results have good generalizability. Furthermore, we employed the MMAS- 8 to define antihypertensive adherence. The MMAS- 8 has been reported in several studies across different cultures and languages as a reliable tool in determining adherence level of patients with HTN $(24,25)$. As mentioned above, we also observed a good reliability and validity of this scale in Persian speaking patients in a previous study (17).

On the other hand, as a broad range of factors can affect medication adherence, we were not able to study all possible related factors such as patient education, psychological factors, attitude and knowledge of the patients, and the like. Also, access to medical records of all patients was not feasible as some were interviewed at pharmacies and some patients were interviewed when they presented to the clinic for the first time and we relied solely on subjective information provided by the patients. Therefore, recall bias might be a concern. However, this was not true in all subjects. 


\subsection{Suggestions for Future Researchers}

It would be a good idea to design a questionnaire to include physical, psychological, therapeutic, and social factors related to healthcare management, and economic issues of the patients who are suffering from another comorbid disease besides HTN when studying medication adherence. As stated earlier, medication adherence is a complex phenomenon and both patient-related factors as well as healthcare system-related factors are involved in it. Studying other important factors, such as medication cost, differences between generic and brand forms of a single medication, visual acuity of patients and the role of the pharmacists seem necessary for better understanding of adherence in Iran. This will be of outmost benefit to have the opportunity for more complex analyses to find factors with significant association with medication adherence.

Although with increasing the number of comorbid conditions, the proportion of patients with high adherence decreases, neither the presence nor the number of comorbid diseases showed statistically significant association with antihypertensive adherence in comorbid hypertensive patients. With individual analysis, neither IHD, nor DM, and nor dyslipidemia showed significant association with antihypertensive adherence.

Appendix 1. The 8-Item Morisky Medication Adherence Scale (MMAS-8)

Item 1 . Do you sometimes forget to take your antihypertensive pills?

Item 2. People sometimes miss taking their medications for reasons other than forgetting. Thinking over the past 2 weeks, were there any days when you did not take your antihypertensive medicine?

Item 3. Have you ever cut back or stopped taking your medication without telling your doctor, because you felt worse when you took it?

Item 4 . When you travel or leave home, do you sometimes forget to bring along your antihypertensive medication?

Item 5. Did you take your antihypertensive medicine yesterday?

Item 6 . When you feel like your antihypertensive is under control, do you sometimes stop taking your medicine?

Item 7. Taking medication every day is a real inconvenience for some people. Do you ever feel hassled about sticking to your antihypertensive treatment plan?

Item 8 . How often do you have difficulty remembering to take all your medications?

Never, rarely, sometimes, usually, or all the time.

\section{Authors' Contributions}

Study concept and design: Yashar Moharramzad; Acquisition of data: Yashar Moharramzad, Zahra Saadat, Mostafa Bahremand, and Hossein Aerab-Sheibani; Analysis and interpretation of data: Yashar Moharramzad and
Habibollah Saadat; Drafting of the manuscript: Farahnza Nikdoust and Elham Shobeiri; Critical revision of the manuscript for important intellectual content: Donald E. Morisky and Mostafa Bahremand; Statistical analysis: Yashar Moharramzad; Administrative, technical, and material support: Zahra Saadat; Study supervision: Habibollah Saadat and Donald E. Morisky.

\section{References}

1. Osterberg L, Blaschke T. Adherence to medication. N Engl J Med. 2005;353(5):487-97.

2. Delamater AM. Improving patient adherence. Clin Diabetes. 2006;24(2):71-7.

3. Carretero OA, Oparil S. Essential hypertension : part II: treatment. Circulation. 2000;101(4):446-53.

4. Krousel-Wood M, Islam T, Muntner P, Holt E, Joyce C, Morisky DE, et al. Association of depression with antihypertensive medication adherence in older adults: cross-sectional and longitudinal findings from CoSMO. Ann Behav Med. 2010;40(3):248-57.

5. Ghembaza MA, Senoussaoui Y, Tani MK, Meguenni K. Impact of patient knowledge of hypertension complications on adherence to antihypertensive therapy. Curr Hypertens Rev. 2014;10(1):41-8.

6. An J, Nichol MB. Multiple medication adherence and its effect on clinical outcomes among patients with comorbid type 2 diabetes and hypertension. Med Care. 2013;51(10):879-87.

7. Briesacher BA, Andrade SE, Fouayzi H, Chan KA. Comparison of drug adherence rates among patients with seven different medical conditions. Pharmacotherapy. 2008;28(4):437-43.

8. Scalco AZ, Scalco MZ, Azul JBS, Lotufo Neto F. Hypertension and depression. Clinics. 2005;60(3):241-50.

9. Natarajan N, Putnam W, Van Aarsen K, Beverley Lawson K, Burge F. Adherence to antihypertensive medications among family practice patients with diabetes mellitus and hypertension. Can Fam Physician. 2013;59(2):e93-e100.

10. Ho PM, Rumsfeld JS, Masoudi FA, McClure DL, Plomondon ME Steiner JF, et al. Effect of medication nonadherence on hospitalization and mortality among patients with diabetes mellitus. Arch Intern Med. 2006;166(17):1836-41.

11. Friedman O, McAlister FA, Yun L, Campbell NR, Tu K, Canadian Hypertension Education Program Outcomes Research T. Antihypertensive drug persistence and compliance among newly treated elderly hypertensives in ontario. Am J Med. 2010;123(2):173-81.

12. Schmittdiel JA, Uratsu CS, Karter AJ, Heisler M, Subramanian U, Mangione CM, et al. Why don't diabetes patients achieve recommended risk factor targets? Poor adherence versus lack of treatment intensification. J Gen Intern Med. 2008;23(5):588-94.

13. Inkster ME, Donnan PT, MacDonald TM, Sullivan FM, Fahey T. Adherence to antihypertensive medication and association with patient and practice factors. J Hum Hypertens. 2006;20(4):295-7.

14. Morisky DE, DiMatteo MR. Improving the measurement of selfreported medication nonadherence: response to authors. J Clin Epidemiol. 2011;64(3):255.

15. Morisky DE, Ang A, Krousel-Wood M, Ward HJ. Predictive validity of a medication adherence measure in an outpatient setting. $J$ Clin Hypertens (Greenwich). 2008;10(5):348-54.

16. Krousel-Wood M, Islam T, Webber LS, Re RN, Morisky DE, Muntne P. New medication adherence scale versus pharmacy fill rates in seniors with hypertension. Am J Manag Care. 2009;15(1):59-66.

17. Moharamzad Y, Saadat H, Nakhjavan Shahraki B, Rai A, Saadat Z, Aerab-Sheibani $\mathrm{H}$, et al. Validation of the Persian Version of the 8-Item Morisky Medication Adherence Scale (MMAS-8) in Iranian Hypertensive Patients. Glob J Health Sci. 2015;7(4):41252.

18. Levey AS, Eckardt KU, Tsukamoto Y, Levin A, Coresh J, Rossert ], et al. Definition and classification of chronic kidney disease: a position statement from Kidney Disease: Improving Global Outcomes (KDIGO). Kidney Int. 2005;67(6):2089-100.

19. Sarayani A, Jahangard-Rafsanjani Z, Hadjibabaie M, Ahmadvand A, Javadi M, Gholami K. A comprehensive review of adherence to diabetes and cardiovascular medications in Iran; implications for practice and research.J Diabetes Metab Disord. 2013;12(1):57. 
20. Chapman RH, Benner JS, Petrilla AA, Tierce JC, Collins SR, Battleman DS, et al. Predictors of adherence with antihypertensive and lipid-lowering therapy. Arch Intern Med. 2005; 165(10):1147-52.

21. Breitscheidel L, Ehlken B, Kostev K, Oberdiek MS, Sandberg A Schmieder RE. Real-life treatment patterns, compliance, persistence, and medication costs in patients with hypertension in Germany.J Med Econ. 2012;15(1):155-65.

22. Lee JK, Grace KA, Taylor AJ. Effect of a pharmacy care program on medication adherence and persistence, blood pressure, and lowdensity lipoprotein cholesterol: a randomized controlled trial. JAMA. 2006;296(21):2563-71.
23. Mant J, McManus RJ. Does it matter whether patients take their antihypertensive medication as prescribed? The complex relationship between adherence and blood pressure control. J Hum Hypertens. 2006;20(8):551-3.

24. Hacihasanoglu Asilar R, Gozum S, Capik C, Morisky DE. Reliability and validity of the Turkish form of the eight-item Morisky medication adherence scale in hypertensive patients. Anadolu Kardiyol Derg. 2014;14(8):692-700.

25. de Oliveira-Filho AD, Morisky DE, Neves SJ, Costa FA, de Lyra DJ. The 8-item Morisky Medication Adherence Scale: validation of a Brazilian-Portuguese version in hypertensive adults. Res Social Adm Pharm. 2014;10(3):554-61. 